\title{
Kosmopolitismus als Großtheorie?
}

\section{Einleitung}

Dem Begriff Kosmopolitismus ist eine gewisse »Sperrigkeit« zu eigen. Dies bemerkt man nicht nur bei dem Versuch, ihn flüssig auszusprechen, sondern auch dann, wenn man aufgefordert wird, ihn kurz und treffend zu definieren.

Ursprünglich in der griechisch-hellenischen Ideenwelt beheimatet und eher als individualistische Lebensphilosphie formuliert, gewann der Kosmopolitismus im 17. und insbesondere im 18. Jahrhundert $u$. a. durch die Werke von Christoph Martin Wieland (siehe dazu u. a. die von ihm 1773-1789 herausgegebene Zeitschrift »Der teutsche Merkur« und Wieland 1804) und Immanuel Kant (1993a [1784], 1993b [1795]) internationalen Geltungsanspruch. Allerdings war er schon damals vor romantisch-visionären Verklärungen nicht gefeit. Ein Zeitgenosse schrieb:

»Der Kosmopolit [...] sucht nur das Wahre, Nützliche, Schöne und Edle, - das allgemeine Ziel der Menschheit - wahre Humanität zu befördern« (Voss 1797, zit. in: Walther 1998).

Der Verdacht, man könnte dem Kosmopolitismus möglicherweise dadurch analytische Schärfe geben, dass man die verschiedenen Ansprüche des Wahren, Nützlichen, Schönen und Edlen getrennt betrachtet, liegt nahe. Dies soll in den folgenden Absätzen mit dem Ziel geschehen, Zweifel an einer Verwendung des Begriffs im Sinne einer Großtheorie zum Ausdruck zu bringen. Großtheorien, die gesamtperspektivisch über das deskriptive und kausale Sein (also über das Wahre), über das normative and ästhetische Sollen (also das Edle und Schöne) sowie über das methodische und politische Tun (also das Nützliche) Bescheid geben, bergen immer zwei Gefahren. Die eine ist die Gefahr der mangelnden Präzision. Eine mehrdimensionale Begriffsverwendung führt nämlich allzu leicht zur Vermengung von Fragen der Ästhetik und des Normativen sowie des Wahren und des Praxeologischen. Dass eine breit angelegte Verwendung des Begriffs Kosmopolitismus genau dieser Gefahr unterliegt, soll in diesem kurzen Beitrag gezeigt werden.

Die andere Gefahr ist die der Ideologisierung. Eine Großtheorie kann dann als ideologisiert gelten, wenn es allerorts notwendig geworden ist, sich zu erklären, ob man Kosmopolit ist oder nicht. Wir wissen nun wirklich nicht, ob wir ein solches Bekenntnis abgeben mögen, zumindest einer der beiden Autoren kann aber nachgewiesenermaßen in Anspruch nehmen, dem kosmopolitischen Anliegen zumindest in Schrift und Wort große Sympathie entgegen zu bringen. Das zeigt sich zunächst in seinen frühen Arbeiten zu internationalen Regimen, in denen die kosmopolitische politische Theorie von Autoren wie Charles Beitz (1979) und Thomas Pogge (1992) explizit als Bezugspunkt zur normativen Bewertung internationaler Regime vorgeschlagen wird (Zürn 1987, 1992a). Parallel zur Entwicklung der Vision einer »kosmopolitischen Weltordnung « von David Held (1995) sind Arbeiten über die Notwendigkeit der Demokratisierung internationaler Institutionen entstanden (Zürn 1992b; 1996, 2000). Die Arbeiten zum Regieren jenseits des Nationalstaates zielten auf die 
Etablierung der These, dass governance im Zeitalter der gesellschaftlichen und politischen Globalisierung eben die Sphäre des Nationalstaates verlassen hat und eine denationalisierte Perspektive zum Verständnis politischer Prozesse notwendig ist (Zürn 1998; Zangl/Zürn 2003; Zürn/Walter 2005; Zürn et al. 2006). In diesem Zusammenhang ist auch explizit der methodologische Nationalismus kritisiert (Zürn 2001) und um bewusst andersartige research designs ergänzt worden (Joerges/Zürn 2005).

Die in diesem Beitrag aufscheinende Kritik kann sich also kaum auf die Grundgedanken des Kosmopolitismus als solches richten. Es geht vielmehr darum zu fragen, ob die Zusammenführung des Wahren, Nützlichen, Schönen und Edlen in einer Großtheorie, wie sie in der Trias der Bände von Ulrich Beck und Edgar Grande (Beck 2002, 2004; Beck/Grande 2004) versucht worden ist, sich als erfolgreich erwiesen hat. Wir verstehen die uns zugewiesene Aufgabe also nicht darin, die Übereinstimmungen der eigenen Arbeiten mit der von Beck und Grande herauszustellen, sondern eine kritische Auseinandersetzung mit dieser Grundkonzeption des Kosmopolitismus vorzunehmen. Diese Kritik soll auch in keinster Weise davon ablenken, dass die Autoren dieses Beitrages in vielfältiger Weise von den Arbeiten von Beck und Grande profitiert haben. Es geht aber um die Frage, ob man sich mit einer solchen begrifflich-konzeptionellen Zusammenführung einer Reihe von unterschiedlichen Diskursen in einem allumfassenden Konzept des Kosmopolitismus nicht allzu weit und ohne Not von den vorhandenen begrifflichen Traditionsbeständen löst - um den Preis der Unschärfe.

\section{Das Schöne und das Edle - neue Vision einer guten politischen Ordnung?}

Beginnen wir mit dem Schönen und Edlen, also den normativen Komponenten des Begriffs. Hier lassen sich eine normative Theorie der guten politischen Ordnung und eine politische Vision unterscheiden. Beck und Grande bemühen sich in dieser Dimension, den Kosmopolitismus als moderne, gleichsam multikulturelle Alternative zum tradierten Universalismus, die aber nicht der Beliebigkeit des Postmodernen unterliegt, zu lokalisieren.

Zentraler Bezugspunkt des universalistischen Denkens ist bis heute Kant, der allerdings in seiner Schrift zum ewigen Frieden (1795) nicht selten schon den Begriff des Kosmopolitischen benutzt hat. Ausgehend von seinen Überlegungen zum Naturzustand, der die Potenz des permanenten Angriffs eines jeden auf einen jeden in sich trägt und deswegen in den »bürgerlich-gesetzlichen Zustand « (Kant 1993 [1795]: 203) überführt werden müsse, benennt er ein bestehendes Beziehungsverhältnis aller Menschen als die zentrale Voraussetzung eines Weltbürgerrechts. ${ }^{1}$

1 »Alle rechtliche Verfassung aber ist, was die Personen betrifft, die darin stehen [...] so fern Menschen und Staaten, in äußerem auf einander einfließendem Verhältnis stehend, als Bürger eines allgemeinen Menschenstaats anzusehen sind (ius cosmopoliticum). Diese Eintheilung ist nicht willkührlich, sondern nothwendig in Beziehung auf die Idee vom ewigen Frieden. Denn wenn nur einer von diesen im Verhältnisse des physischen Einflusses auf den andern, und doch im Naturstande wäre, so würde damit der Zustand des Krieges verbunden seyn, von dem befreyet zu werden hier eben die Absicht ist« (Kant 1993 [1795]: 203). 
Damit gründet er das Weltbürgerrecht auf drei Einsichten: Einmal auf die Anerkennung eines universalistischen Gleichheitsprinzips und der moralischen Autonomie des Individuums, zum Zweiten auf die Einsicht in die Notwendigkeit seiner rechtsstaatlichen, prozeduralen Verankerung und zum Dritten auf die Gleichsetzung des (von positivem Recht noch nicht berührten) Raums des Naturzustandes mit dem Zustand des ewigen Krieges.

Wie unterscheiden sich nun Beck und Grande von diesen Grundlegungen? Was ist in normativer Hinsicht neu am Kosmopolitismus? Kosmopolitismus bedeutet für sie »eine besondere Form des gesellschaftlichen Umgangs mit kultureller Andersartigkeit« (Beck/Grande 2004: 25). Im Gegensatz zum Universalismus, der entweder substanziell oder prozedural Andersartigkeit auflöse, zeichnet sich der Kosmopolitismus dadurch aus,

»dass hier im Denken, Zusammenleben und Handeln die Anerkennung von Andersheit zur Maxime wird [...]. Der Kosmopolitismus bejaht, was in den beiden Positionen der hierarchischen Verschiedenheit und der universellen Gleichheit ausgeschlossen ist: die Anderen als verschieden und als gleich wahrzunehmen« (Beck/Grande 2004: 27).

Dem Universalismus wird hier die unabwendbare Tendenz zur hegemonialen Entfaltung zugeschrieben (siehe dazu auch Beck 2004: 78-85) - und damit wird er abgelehnt. Andererseits wird er aber mit der Einsicht in die Notwendigkeit, »einen gewissen Bestand an universellen Normen, die es ermöglichen, den Umgang mit Andersartigkeit zu regulieren« (Beck/Grande, 2004: 31) zu sichern, wieder ins Spiel gebracht. Letzteres geschieht in dem Bemühen, den Kosmopolitismus vom Relativismus bzw. von der postmodernen Beliebigkeit des »Alles so schön bunt hier« abzugrenzen. Diese Abgrenzung geschieht aber dadurch, dass der Kosmopolitismus sich als undeutliche Variante des Universalismus zu erkennen gibt - denn die oben unterstellte Unfähigkeit des Universalismus, Andersartigkeit (an)zu erkennen, besteht weder logisch noch empirisch. Ein prozeduraler Universalismus setzt Andersartigkeit notwendig voraus, sonst wäre er schlicht überflüssig. ${ }^{2}$

Die normative Neuigkeit des Kosmopolitismus scheint sich somit zu beschränken auf seine prinzipielle Fundierung:

»Während Universalismus und Nationalismus [...] auf dem Prinzip des Entweder-Oder basieren, beruht der Kosmopolitismus auf dem Prinzip des Sowohl-als-Auch. Das Fremde wird nicht als bedrohlich, desintegrierend, fragmentierend, sondern als bereichernd erfahren und bewertet. Es ist die Neugierde auf mich selbst und das Anderssein, die die Anderen für mich unersetzbar macht. Es gibt einen Egoismus des kosmopolitischen Interesses. Wer die Sicht der Anderen im eigenen Lebenszusammenhang integriert, erfährt mehr über sich selbst und den Anderen« (Beck/Grande 2004: 27f).

Abgesehen davon, dass hier sprachlogisch nur das »sowohl« ausgebreitet wird und das »als auch« unter den Tisch fällt, wird das dem Universalismus unterstellte »Entweder-Oder« von den Autoren in ihrer Abgrenzung zum Relativismus ein paar

2 Aber ein prozeduraler Universalismus setzt ebenfalls eine grundlegende Definition von Gleichheit voraus (ansonsten könnten Zugangs- und Geltungsbedingungen der Prozeduren nicht formuliert werden), die ihrerseits nicht prozedural begründet sein kann, sondern substanziell legitimiert werden muss. Eine solche Grundlegung ist die Annahme der grundsätzlichen Gleichheit aller Menschen. 
Seiten später auch für den Kosmopolitismus entdeckt. ${ }^{3}$ Zudem überrascht doch das naiv-optimistische Menschenbild, das diesen Zeilen zugrunde zu liegen scheint, angesichts der Rückzugsgefechte, die der reine Multikulturalismus derzeit leisten muss.

Im Ergebnis scheint die von Beck und Grande angebotene und nur schattenhaft umrissene Ordnungsvorstellung im Wesentlichen eine universalistische zu sein, ihre Grundlegung dagegen lässt eine revolutionäre Errungenschaft des 18. Jahrhundert einfach unter den Tisch fallen: nämlich die, dass der Mensch in all seiner Verschiedenheit ein Mensch ist und durch dieses Menschsein unveräußerliche Rechte hat, die schützenswert sind. Damit teilen sie nur die zweite von Kants Einsichten, nämlich die Notwendigkeit der prozeduralen Verankerung von (universalistischen) Normen. Diese zu formulieren, dürfte ihnen jedoch ohne die erste Einsicht in das universelle Gleichheitsprinzip schwerfallen und ihre Notwendigkeit können sie ohne ein etwas pessimistischeres Bild der menschlichen Natur, wie in Kants dritter Einsicht in die Qualität des Naturzustandes formuliert, auch nicht begründen. Damit scheint das Neue an dieser Dimension des Kosmopolitismus schwer begründbar und konzeptionell unausgereift. Die theoretischen Debatten über das Verhältnis von kulturellen Rechten und individuellen Grundrechten, wie sie in der politischen Theorie zwischen Liberalen und Republikanern oder der philosophischen Debatte um Menschenrechte im interkulturellen Kontext (vgl. dazu Paul 2001) geführt werden, scheinen uns bei den hier aufgeworfenen Fragen ein gewichtiger Anknüpfungspunkt, der besser nicht ignoriert werden sollte.

\section{Das Nützliche - Kritik des methodologischen Nationalismus}

Kommen wir nun zum Nützlichen des Kosmopolitismus, also seiner methodologischen Wendung und seinen Konsequenzen für die institutionelle Gestaltung einer postnationalen politischen Ordnung. Der methodologische Kosmopolitismus erscheint hier zunächst einmal als Kritik am methodologischen Nationalismus:

»Die Zombie-Wissenschaft des nationalen Blicks, die in Kategorien von internationalem Handel, internationalem Dialog, nationaler Souveränität, nationalen Gemeinschaften, dem >Staatsvolk < etc. denkt und forscht, wird zur Unwirklichkeitswissenschaft einer $>$ Nationalsoziologie<: Ebenso wie die Nationalökonomie ist die Nationalsoziologie am Ende ihres Lateins. Denn verkannt wird und unerforscht bleibt, in welchem Ausmaß bereits transnationale Lebensformen, Transmigranten, globale Eliten, supranationale Organisationen und Dynamiken die Verhältnisse in und zwischen den nationalstaatlichen Machtbehältern bestimmen« (Beck 2002: 52).

3 »Der realistische Kosmopolitismus setzt ein universalistisches Minimum voraus. Dazu gehören inhaltliche Normen, die auf keinen Fall verletzt werden dürfen: daß Kinder, Frauen nicht verkauft, nicht versklavt werden dürfen und daß jedermann aussprechen darf, was er von Gott und seiner Regierung hält, ohne gefoltert und mit dem Tode bedroht zu werden, ist eine solche Selbstverständlichkeit, daß ein Verstoß dagegen nicht auf kosmopolitische Toleranz stoßen darf« (Beck 2004: 77f; siehe auch Beck/Grande 2004: 31). 
Ausgehend von einer reichhaltigen Bestandsaufnahme von Denationalisierungstendenzen und der allgemeinen Einsicht, »dass politisches, ökonomisches und kulturelles Handeln samt seinen (gewussten und ungewussten) Folgen kein[e] Grenzen kennen [...] kann« (Beck 2004: 31), wird ein kosmopolitischer Blick gefordert, der nicht mehr Nationalstaaten und ihre Grenzen privilegiert in den Blick nimmt. Damit wird eine sicherlich notwendige Adaption der sozialwissenschaftlichen Forschung an sich dem nationalistischen Blick nur schwer erschließende Forschungsfelder wie z. B. Europa gefordert. Die Charakteristika des Systems internationaler Beziehungen sollen nicht mehr nur über die Analyse der Interaktionen von Nationalstaaten erschlossen werden, sondern die nationalstaatlichen Strukturen werden (mindestens auch) analysiert als Folgewirkungen der Struktur transnationaler und internationaler Beziehungen (vgl. z. B. zu 2nd image reversed Ansätzen Gourevitch 1978). Unabhängig von den Erfolgsaussichten kosmopolitischen Handelns in der Politik soll nach Becks Ansicht der methodologische Kosmopolitismus als »Neue Kritische Theorie in kosmopolitischer Absicht « durchgesetzt werden, um den oben angesprochenen Defiziten abzuhelfen (Beck 2002: 54).

Praxeologisch wird eine Diskursstrategie empfohlen, die transnationale gesellschaftliche, vor allem aber staatliche Gegenmacht zum globalen Kapital erzeugt. Die staatliche Gegenmacht kann sich allerdings erst dann transnational konfigurieren, wenn Transnationalisierung nicht mehr als Verlust verstanden, sondern als Gewinnchance genutzt wird:

»Für die bisher unterschiedenen Neuformen der Staatlichkeit - den neoliberalen und den ethnischen Staat - gilt übereinstimmend nicht, was auf die folgenden zwei Staatsformen - den transnationalen Überwachungsstaat und den kosmopolitischen Staat - zutrifft: Sie nutzen nicht die Machtchancen, die die kooperative transnationale Souveränität eröffnet« (Beck 2002: 382).

Hinsichtlich der institutionellen Ausgestaltung des kosmopolitischen Staates wird man hier allerdings nicht fündig (Beck 2002: 382f), hinsichtlich der neuen Figur der Europäischen Integration liefern Beck und Grande dann 2004 die Formel vom »Imperium ohne Imperator « (Beck/Grande 2004: 119), das gekennzeichnet sei durch ein institutionelles Mehrebenensystem, eine relativ schwache Parlamentarisierung und eine an Konsens und Kooperation orientierte Funktionsweise (Beck/ Grande 2004: 120-122).

In der methodologischen Kritik des nationalen Blicks scheint dennoch tatsächlich ein Mehrwert des Kosmopolitismus erkennbar zu sein. Momentan bleibt dieser aber noch zu sehr im Unterfangen der Kritik stecken und muss sicher noch weiterentwickelt werden, um als eigener und alternativer Zugriff zum methodologischen Nationalismus bestehen zu können. Die aus dem kosmopolitischen Blick abgeleiteten institutionellen Charakteristika des neuen Staates oder Staatenzusammenschlusses, der da seiner Analyse harrt, können dagegen nicht so recht überraschen. In beiden Fällen würde die Entwicklung der eigenen Position in Weiterführung der bestehenden Überlegungen den geneigten Lesern es viel einfacher machen, das tatsächlich Neue kritisch zu rezipieren. Der Anspruch, etwas komplett Neues in Abgrenzung von ganzen Disziplinen anzubieten, ist nicht sachdienlich. Sowohl der Bestand an Methodik der Analyse transnationaler und internationaler Beziehungen als auch die 
vorhandenen praxeologischen Überlegungen zu global governance und zum Regieren in komplexen Mehrebenensystemen müssten einfach systematisch berücksichtigt werden.

Ein weiterer caveat soll hier nicht unerwähnt bleiben: Gerade ein methodisch strikt kosmopolitischer Blick mag zu politischen Empfehlungen führen, die zur Rückbesinnung auf den Nationalstaat führen (wie Beck auch selbst vermutet, siehe z. B. Beck 2002: 31). Viele der neueren Analysen etwa zur Internet Corporation for Assigned Names and Numbers (ICANN), aber auch zum Scheitern der humanitären Interventionen tendieren genau dazu. Es ist hier nicht die Frage, ob solche Analysen richtig liegen. Sie verweisen allerdings auf den Tatbestand, dass eine ergebnisoffene (d. h. kritische) Sozialwissenschaft niemals auf einen unmittelbaren Zusammenhang zwischen Methode und politischer Empfehlung setzen sollte. Deswegen erscheint uns die Zusammenführung des Wahren, Nützlichen, Edlen und Schönen unter einem begrifflichen Dach so problematisch. Der Zwang, Normatives, Deskriptives und Praxeologisches als eng zusammenhängend, gleichsam als eins zu denken, birgt die Gefahr der Ideologisierung. Diese Gefahr thematisiert Beck (2004: 70-75) auch, es bleibt aber die Frage, ob das schiere Konzept des Kosmopolitismus es nicht zwingend in sich trägt.

\section{Das Wahre-Konkurrierende Befunde}

Kommen wir nun zu guter Letzt zum Wahren, das der Kosmopolitismus will, also zu seinem analytisch-konzeptionellen Charakter. Ausgehend von der Kritik am methodologischen Nationalismus und der Beobachtung einer massiven Zunahme grenzüberschreitender Transaktionen finden sich in dieser Bedeutung des Konzepts eine Reihe interessanter Einsichten und Hinweise. Sicherlich wäre eine systematischere Entfaltung der Hypothesen und Befunde wünschenswert. Störend finden wir aber vor allem, dass auf den inzwischen doch reichhaltigen Literaturbestand zu diesen Fragen zwar verwiesen wird, aber eben keine systematische Auseinandersetzung und Weiterentwicklung mit vorhandenen Konzepten und Wissensbeständen erfolgt. Worin unterscheidet sich das Gesagte von dem Wissensbestand, den wir inzwischen zu global governance und der Rolle von NGOs darin haben? Berichten Beck und Grande eigentlich über eine Weltgesellschaft im Sinne Luhmanns oder über die Formierung lokaler Gesellschaften durch Weltkultur (vgl. Meyer et al. 1997)? Andere Anknüpfungspunkte, an die eben nicht angeknüpft wird, sind die Globalisierung von Kommunikationsräumen und der Verlust operativer Geschlossenheit nationaler Gesellschaften (vgl. Luhmann 1975; Stichweh 2000) oder die Neuordnung von Zeit und Raum als Folge von Denationalisierung (vgl. Sassen 2000, 2006).

Kosmopolitismus in Beckscher Fassung will alle hier kurz benannten Phänomene zusammenfassen und gerade dadurch etwas ganz Neues bieten. Dafür fehlt aber ein konzeptioneller Rahmen - es ist auch nicht so recht ersichtlich, wie dieser aussehen könnte und welchen analytischen Gewinn eine Zusammenfassung unter der Überschrift Kosmopolitismus erbringen könnte. 


\section{Fazit: Kosmopolitismus - Ambitionen und Erträge}

Zusammenfassend scheinen sich hinter dem Begriff des Kosmopolitismus normative, visionäre, methodologische und analytische Aspekte $\mathrm{zu}$ verbergen, deren Zusammenführung kaum einen Zugewinn erbringt, aber Folgeprobleme schafft. Eine Analyse der Aspekte des Begriffs, wie sie hier in der gebotenen Kürze vorgenommen wurde, konnte sicherlich im Einzelnen einen Mehrwert erkennen lassen. Für die konzeptionelle Dimension gilt, dass die Zusammenführung mit einem erheblichen Verlust an analytischer Trennschärfe einhergeht, weil der Begriff zu viele Phänomene in sich vereinen will. In der normativen Dimension versucht er aus einer ebenso kurzen wie gewagten Reduzierung anderer normativer Konzepte sich selbst zu entwerfen und landet dann bei einer verstümmelten und dysfunktionalen Variante eines der verworfenen Prinzipien.

Die Analyse jedweden »über«-nationalen Zusammenhangs wird mit Sicherheit von einem kosmopolitisch geschärften Blick profitieren, darüber hinaus muss die Ergiebigkeit des Kosmopolitismus jedoch erst noch bewiesen werden - und zwar in produktiver Auseinandersetzung mit bekannten Konzepten und Befunden und durch eine Begründung, weshalb es im postideologischen Zeitalter wissenschaftlich sinnvoll sein soll, das Wahre, Nützliche, Schöne und Edle in einen Begriff packen zu wollen.

\section{Literatur}

Beck, Ulrich 2002: Macht und Gegenmacht im globalisierten Zeitalter. Neue weltpolitische Ökonomie, Frankfurt a. M.

Beck, Ulrich 2004: Der kosmopolitische Blick oder: Krieg ist Frieden, Frankfurt a. M.

Beck, Ulrich/Grande, Edgar 2004: Das kosmopolitische Europa. Gesellschaft und Politik in der Zweiten Moderne, Frankfurt a. M.

Beitz, Charles R. 1979: Political Theory and International Relations, Princeton, NJ.

Gourevitch, Peter 1978: The Second Image Reversed: The International Sources of Domestic Politics, in: International Organization 32: 4, 881-912.

Held, David 1995: Democracy and the Global Order: From the Modern State to Cosmopolitan Governance, Cambridge.

Joerges, Christian/Zürn, Michael (Hrsg.) 2005: Law and Governance in Postnational Constellations: Compliance Beyond the Nation-State, Cambridge.

Kant, Immanuel 1993a (1784): Ideen zu einer allgemeinen Geschichte in weltbürgerlicher Absicht, in: Kant, Immanuel: Schriften zur Anthropologie, Geschichtsphilosophie, Politik und Pädagogik (Werkausgabe Band XI, herausgegeben von Wilhelm Weischedel), Frankfurt a. M., 33-50.

Kant, Immanuel 1993b (1795): Zum ewigen Frieden. Ein philosophischer Entwurf. in: Kant, Immanuel: Schriften zur Anthropologie, Geschichtsphilosphie, Politik und Pädagogik (Werkausgabe Band XI, herausgegeben von Wilhelm Weischedel), Frankfurt a. M., 191251.

Luhmann, Niklas 1975: Die Weltgesellschaft, in: Luhmann, Niklas: Soziologische Aufklärung, Band 2: Beiträge zur funktionalen Differenzierung der Gesellschaft, Opladen, 51-71.

Meyer, John W./Boli, John/Thomas, George M./Ramirez, Francisco O. 1997: World Society and the Nation-State, in: American Journal of Sociology 103: 1, 144-181. 
Paul, Gregor 2001: Philosophie der Menschenrechte. Ergebnisse eines Projekts, in: http:// agd.polylog.org/3/ppg-de.htm; 6.10.2006.

Pogge, Thomas W. 1992: Cosmopolitanism and Sovereignty, in: Ethics 103: 1: 48-75.

Sassen, Saskia 2000: Machtbeben. Wohin führt die Globalisierung?, Stuttgart.

Sassen, Saskia 2006: Territory, Authority, Rights: From Medieval to Global Assemblages, Princeton.

Stichweh, Rudolf 2000: Die Weltgesellschaft. Soziologische Analysen, Frankfurt a. M.

Walther, Rudolph 1998: Weltbürger, gebt den Staat nicht auf!, in: http://www.zeit.de/1998/31/ 199831.t global.xml; 5.10.2006.

Wieland, Christoph Martin 1804: Das Geheimnis des Kosmopoliten-Ordens, Wien.

Zürn, Michael 1987: Gerechte internationale Regime. Bedingungen und Restriktionen der Entstehung nicht-hegemonialer internationaler Regime untersucht am Beispiel der Weltkommunikationsordnung, Frankfurt a. M

Zürn, Michael 1992a: Interessen und Institutionen in der internationalen Politik. Grundlegung und Anwendungen des situationsstrukturellen Ansatzes, Opladen.

Zürn, Michael 1992b: Jenseits der Staatlichkeit, in: Leviathan 20: 4, 490-513.

Zürn, Michael, 1996: Über den Staat und die Demokratie im europäischen Mehrebenensystem, in: Politische Vierteljahresschrift 37: 1, 27-55.

Zürn, Michael 1998: Regieren jenseits des Nationalstaates. Globalisierung und Denationalisierung als Chance, Frankfurt a. M.

Zürn, Michael, 2000: Democratic Governance Beyond the Nation-State: The EU and Other International Institutions, in: European Journal of International Relations 6: 2, 183-221.

Zürn, Michael 2001: Politik in der postnationalen Konstellation. Über das Elend des methodologischen Nationalismus, in: Landfried, Christine (Hrsg.): Politik in einer entgrenzten Welt (Tagungsband des 21. Wissenschaftlichen Kongresses der Deutschen Vereinigung für Politische Wissenschaft), Köln, 181-203.

Zürn, Michael/Binder, Martin/Ecker-Ehrhardt, Matthias/Radtke, Katrin 2006: Politische Ordnungsbildung wider Willen. Ein Forschungsprogramm zu transnationalen Konflikten und Institutionen (WZB Discussion Paper IV 2006-301), Berlin.

Zürn, Michael/Walter, Gregor (Hrsg.) 2005: Globalizing Interests: Pressure Groups and Denationalization, Albany, NY.

Zangl, Bernhard/Zürn, Michael 2003: Frieden und Krieg. Sicherheit in der nationalen und post-nationalen Konstellation, Frankfurt a. M. 